certain experimental conditions. If $M$ is a solvent molecule, the energy may be dissipated by consecutive collisions, but it may also be attached to one monomeric particle in the course of reproduction of a germ with the aid of the solvent. In recent papers of Dostal, Flory, Mark, Schulz and others, these various possibilities are discussed and brought into relation with the experimental facts.

The result is that at present no polymerization process can yet be resolved quite clearly into all its elementary steps, but there is no doubt that the three above-mentioned processes, namely, chain start, chain growth, and chain ending always take the outstanding role during a polymerization reaction. Increasing experimental material will enable us to separate more and more neatly the different possible steps and to work out something like a fine structure of polymerization reactions.

\section{Polycondensation Reactions}

This is the second type of reaction by which high polymers can be produced. Here also we will confine ourselves to reactions which lead to pure chain polymers. In a condensation reaction a new molecule is built by removal of a part of the reactant particles; this part is usually water. If a dibasic acid, for example, succinic acid, reacts with glycol one gets :

$$
\begin{gathered}
\mathrm{HO} \cdot \mathrm{H}_{2} \mathrm{C}-\mathrm{CH}_{2} \cdot \mathrm{OH}+\mathrm{HOOC} \cdot \mathrm{CH}_{2} \cdot \mathrm{CH}_{2} \cdot \mathrm{COOH} \rightarrow \\
\mathrm{HOH}_{2} \mathrm{C} \cdot \mathrm{CH}_{2} \mathrm{O} \cdot \mathrm{OC} \cdot \mathrm{CH}_{2} \cdot \mathrm{CH}_{2} \cdot \mathrm{COOH}+\mathrm{H}_{2} \mathrm{O} \quad(e)
\end{gathered}
$$

This esterification leads to a molecule which can again react with an alcohol or with an acid, producing further reactive molecules and growing thus slowly to a long chain with alternate alcohol and acid members.

Reastions like this have been investigated recently by Dostal, Flory, Marecek, Raff and others. They are much easier to deal with because the intermediate products are not unsaturated but of the same type as the monomeric substance. It is therefore not a chain reaction, which we have before us, but a stepwise esterification for which kinetic formulæ can be derived without serious difficulties. The problem of the mutual interaction of three different elementary processes as it has to be solved in the case of polymerization is here reduced to the mathematical study of a step reaction with many steps. The production of 'germs' is here of the same order of magnitude as the velocity of growth, and therefore the chains which are formed are much shorter than in the case of polymerization. Therefore a fairly good agreement was obtained by comparing the results of experiments with the above-mentioned formulæ, and the problem of polycondensation reactions does not offer serious difficulties to a quantitative understanding.

This is a reason why the efforts of workers are being more and more concentrated on the study of polymerization processes, which still offer a great number of unsolved and interesting problems.

\title{
The Solar Eclipse of June 19, 1936
}

A $\mathrm{N}$ old practice was revived on May 27, when a joint meeting of the Royal Society and the Royal Astronomical Society was held to receive reports on the expeditions organized by the Joint Permanent Eclipse Committee of the two societies for the total solar eclipse of June 19, 1936. It was sad to observe that on this occasion the tradition by which the president of the Royal Astronomical Society sat facing the audience alongside the president of the Royal Society was allowed to lapse.

Prof. F. J. M. Stratton opened the discussion with an account of the expedition to Kamishari in Hokkaido, staffed from the Solar Physics Observatory, Cambridge, with the addition of Dr. T. Royds, of the Kodaikanal Observatory, sent by the Government of India. $\mathrm{He}$ mentioned that the site was chosen after consultation with the Meteorological Office and consideration of the data supplied by the National Research Council of
Japan. Unfortunately, the prevailing wind which should have given the desired weather for the eclipse chose the wrong week to prevail, and the day of the eclipse was cloudy. The second half of the partial phase was happily clear, and Dr. Royds was enabled to carry through most of his programme successfully; second contact was observed through gathering elouds which spoilt by scattering light the programme of observing intensities of chromospheric spectral lines at different heights above the sun's limb, though flash spectra were obtained; within five seconds of the commencement of totality the sun was completely covered by cloud too thick even for the infra-red cameras to penetrate; the sun did not emerge into a clear sky until 10 minutes after the end of totality.

It was unfortunate that the half of the eclipsed sun which was in clear sky for second contact and for the first few seconds of totality was on the 
opposite limb to that of second contact, and valuable observations were lost by a margin of seconds only in the race of moon and clouds to cover the sun. Prof. Stratton devoted his remarks to the part of the programme which was lost due to the clouds-the polarization of the coronal light to be observed by Dr. C. W. Allen with a double camera and a Nicol prism in front of one lens, and the direction of polarization of skylight in the neighbourhood of the eclipsed sun, to be observed photographically by himself and visually by Dr. F. W. Aston with a set of Nicol prisms and Savart plates. Prof. Stratton paid a tribute to the generous help and assistance received from the Japanese authorities and scientific colleagues, and mentioned that, of the three neighbouring Japanese expeditions, all of whom had a clear sky for the eclipse, one was led by Prof. Matsukuma, of Sendai University, who had stood down from his original plan to go to Kamishari to make room for the English party.

Dr. Royds next gave an account of his measures of the displacement of the Fraunhofer lines at the centre and limb of the sun's disk with and without an eclipse. His values were as follows:

\begin{tabular}{ccccc} 
& \multicolumn{4}{c}{ Mean displacement to red } \\
Intensity & \multicolumn{2}{c}{ Without eclipse } & During eclipse \\
of lines & Centre & Limb & Centre & Limb \\
& A. & A. & A. & A. \\
11.0 & 0.0100 & 0.0122 & 0.0078 & 0.0126 \\
5.2 & 0.0084 & 0.0148 & 0.0088 & 0.0151 \\
3.0 & 0.0060 & 0.0122 & 0.0058 & 0.0116
\end{tabular}

It was clear from Dr. Royds' eclipse observations that the values obtained from the limb without eclipse had not been seriously affected by light scattered from the bright centre of the disk, which would especially affect the weak lines. Observations had been made at distances from the centre of the sun's disk of $0,0.28,0.57,0.76,0.90,0.95$, 0.97 times the sun's radius: the observation at 0.992 and the hoped-for observation of chromospheric lines were lost owing to clouds. The explanation in terms of convection currents for lines of different intensities originating at different levels in the sun, which had been offered for the discrepancies at the centre of the sun, could not hold for the limb, and the divergencies from the predicted Einstein displacements had yet to be explained.

Dr. R. O. Redman discussed the advantages from spectrophotometry of the chromosphere of a jumping slit spectrograph (taking a series of exposures with a fixed plate) over a continuously moving slitless spectrograph: there was less trouble from scattered light, higher resolution, greater purity of spectrum, and no trouble such as arises from the varying width of the steadily narrowing crescent photographed with the slitless instrument. He described the moving back camera specially designed by Mr. C. R. Davidson and himself for the eclipse, the instrumental setup, and the programme of exposures at intervals of one second carried out at the eclipse through second contact. As defined by the final disappearance of the Fraunhofer spectrum, totality commenced 1 second late on the computed time.

Dr. A. D. Thackeray gave an account of the flash spectra secured by Dr. Redman with the moving plate camera and by himself with the Hills quartz spectrograph fed by light from an aluminized mirror. While in both cases the original plan of observing the changes of intensity of spectral lines with solar heights had to be abandoned because of light scattering by the clouds, yet much of value could be learned from the plates. In particular, Dr. Redman's spectra included one of the finest for the study of the transition from absorption to emission spectra that had ever been secured. He directed attention to the following points of special interest: $(a)$ the presence of a bright line at $3969 \cdot 40 \mathrm{~A}$. between $H \varepsilon$ and $[\mathrm{H}]$ never previously recorded in flash spectra and evidence of the excellent definition secured by Dr. Redman; (b) the presence in absorption of the subordinate series $3^{2} P^{0}-n^{2} S$ of $\mathrm{Na}$ at a stage where the $D$ lines had practically vanished in transition from absorption to emission; (c) the displacement towards the violet of emission lines compared with the absorption lines in a spectrum taken at a distance $09994 r$ from the centre of the sun's disk; (d) the gradient of the Balmer series in emission from $\mathrm{H} \beta$ to $\mathrm{H} 26$, with a value of lower weight for the total emission of $\mathrm{H} \alpha$ : the gradient showed manifest departure from the values given by the Schrödinger-Pauli formula, as had been found by Davidson and Stratton in the 1926 eclipse.

Prof. J. A. Carroll described the equipment of the expedition from Aberdeen to Omsk : the objective interferometer prepared for a monochromatic image of the whole corona crossed by interference rings which should show by their displacements internal movements in the corona; a highdispersion echelon spectroscope with 35 plates immersed in fluid and giving an effective thickness to each plate of $1 \mathrm{~mm}$. in air ; and a spectrograph for the extreme infra-red spectra of the corona and chromosphere. He then dealt in detail with the method of reducing the daily range of temperature of $12^{\circ} \mathrm{C}$. to the range that could be allowed without loss of definition for the instruments, $0.1^{\circ}$ for lenses and prisms, $0.01^{\circ}$ for the interferometer and $0.001^{\circ}$ for the echelon. Large thermal inertia in the optical parts and small and slow changes in the surround of each instrument were required. The hut was doubled-walled and 
lagged inside; it was also equipped with heating and refrigerating plant controlled by thermostats and by fans for stirring the atmosphere. The instruments in their turn were surrounded by a further lagged cover with again a thermostatically controlled heating circuit inside. This first serious attempt at delicate temperature control under eclipse camp conditions had worked very successfully.

Mr. E. G. Williams spoke of the results obtained with the infra-red spectrograph. A composite plate had been used of special rapid panchromatic for 5800-6700 A., Agfa 800 for the 8000 region and Agfa 950 for the region beyond $9000 \mathrm{~A}$. It had been intended to use Agfa 1050 for the region beyond 10,000 A., but though the plates were specially flown over from Berlin to Moscow they were no longer sensitive when the day of eclipse arrived and could not be used. They were, therefore, unable to secure the lines at $10,746 \cdot 80 \mathrm{~A}$., 10,797 95 A. recently reported by Iyot. They did get a line at $7591 \cdot 3 \mathrm{~A}$. in the coronal spectrum for which Curtis and Babcock in 1928 gave the wave-length $7896 \mathrm{~A}$. and lyot more recently from the Pic du Midi gave 7891.9 A. No trace was found of the line about $9609 \mathrm{~A}$. to be expected if the coronal spectrum came from a doubly excited helium atom. In the chromospheric spectrum lines of the Paschen series were obtained, for the first time at an eclipse, from $P 4$ to P17, and other lines were identified as due to $\mathrm{He}, \mathrm{O}$, $\mathrm{Mg}$ and $\mathrm{Ca}^{+}$.

In the subsequent discussion Mr. J. Evershed, referring to Dr. Royds' excellent spectra, pointed out that to reduce his shifts from an are in air to an arc in vacuo meant an increase of $0.003 \mathrm{~A}$. and meant that the displacement at the limb was twice the predicted Einstein value. Prof. A. Fowler, in sympathizing with the Cambridge party on their failure, said that it was a magnificent failure. He was very much impressed by the mechanism of Dr. Redman's camera and with the very interesting and beautiful results he had obtained. $\mathrm{He}$ would also like to congratulate Mr. Williams on his infra-red spectra.

\section{Obituary}

Prof. A. G. Perkin, F.R.S.

$\mathrm{P}^{\mathrm{p}}$ ROF. A. G. PERKIN died at his Leeds residence on May 30. Born at Sudbury, Middlesex, in December 1861, he was the second son of the late Sir William Perkin, brother of the late W. H. Perkin, jun., Waynflete professor of chemistry in the University of Oxford, and half-brother of the late Dr. F. M. Perkin, who was well known as a consulting chemist.

A. G. Perkin grew up in an atmosphere of chemistry and zeal for scientific investigation. His education was varied : he followed in his father's footsteps by attending the City of London School, and from 1877 until 1879 studied under Frankland and Guthrie at the Royal College of Chemistry, South Kensington, where he carried out the investigation leading to his first paper, "The Action of Nitric Acid on Di-ptolylguanidine", communicated to the Chemical Society in 1880. He next spent a year at Anderson's College, Glasgow, under E. J. Mills, and finally a year in the Dyeing Department, Yorkshire College, Leeds, where he worked with J. J. Hummel on new compounds derived from the colouring matters of brazilwood and logwood. Perkin always paid generous tribute to the abilities and personality of Prof. Hummel, to whom he owed his first, and lasting, enthusiasm for the study of the natural colouring matters.

In 1882 Perkin left the Yorkshire College to take up an appointment as chemist at the alizarin factory of Hardman and Holden, Ltd., Manchester, and was promoted to the position of manager in 1888. During this commercial period he continued scientific investigations and published papers on the action of nitric acid on anthracene, and in collaboration with W. H. Perkin, jun., on derivatives of anthraquinone, and on the colouring matter of the Indian dyestuff, kamala.

Perkin resigned his position with Hardman and Holden, Ltd., in 1892 to join the staff of the Dyeing Department, Yorkshire College, as lecturer and research chemist. Then followed a period of more than twenty years of intensive research, mainly concerned with the isolation of the colouring principles of natural products and the investigation of their constitutions. The profound knowledge of natural colouring matters that he gained by his brilliant researches established his international reputation in this field. He examined numerous natural colouring matters by degradative methods, and in certain instances deduced their constitutions, many of which, for example, those of catechin, luteolin, gossypetin, quercetagetin, etc., have since been confirmed synthetically by other workers.

In later years, Perkin devoted more of his time to the chemistry of anthraquinone derivatives. A study of the migration of the acyl group in partially acylated phenolic compounds led to the synthesis of, inter alia, some hydroxyanthraquinone methyl ethers, originally isolated from the Indian natural dyestuff, chay root, but not hitherto obtained synthetically. The constitutions of numerous hydroxyanthranols were: 